CERN-TH/96-146

FERMILAB-conf/96-129-T

hep-ph/9606209

\title{
W PLUS HEAVY QUARK PRODUCTION AT THE TEVATRON
}

\author{
Walter T. Giele, Stephane Keller \\ Fermilab, MS 106 \\ Batavia, IL 60510, USA \\ and \\ Eric Laenen \\ CERN TH-Division \\ 1211-CH, Geneva 23, Switzerland
}

\begin{abstract}
We present the next-to-leading order QCD corrections to the production of a $W$-boson in association with a jet containing a heavy quark. The calculation is fully differential in the final state particle momenta and includes the mass of the heavy quark. We study for the case of the Tevatron the sensitivity of the cross section to the strange quark distribution function, the dependence of the cross section on the heavy quark mass, the transverse momentum distribution of the jet containing the heavy quark, and the momentum distribution of the heavy quark in the jet.
\end{abstract}

To appear in:

Proceedings of the DESY-Zeuthen Workshop: "QCD and QED in Higher Orders", Rheinsberg, Germany, April 1996.

CERN-TH/96-146

FERMILAB-conf/96-129-T

June 1996 


\title{
W plus heavy quark production at the Tevatron *
}

\author{
Walter T. Giele, Stephane Keller ${ }^{\mathrm{a}}$ and Eric Laenen ${ }^{\mathrm{b}}$

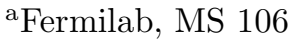 \\ Batavia, IL 60510, USA \\ ${ }^{\mathrm{b}}$ CERN TH-Division \\ CH-1211 Geneva 23, Switzerland
}

We present the next-to-leading order QCD corrections to the production of a $W$-boson in association with a jet containing a heavy quark. The calculation is fully differential in the final state particle momenta and includes the mass of the heavy quark. We study for the case of the Tevatron the sensitivity of the cross section to the strange quark distribution function, the dependence of the cross section on the heavy quark mass, the transverse momentum distribution of the jet containing the heavy quark, and the momentum distribution of the heavy quark in the jet.

\section{INTRODUCTION}

The study of jet production in association with a vector boson at hadron colliders has been succesful in the recent past. The advantage of this signal over pure jet production is that the lepton(s) from the vector boson decay can be used as a trigger, such that jets can be studied free from jet-trigger bias. Furthermore, the lower rate obviates the need for prescaling. On the theory side, progress in calculational techniques to construct next-to-leading order (NLO) Monte-Carlo programs [1] has allowed a meaningful confrontation with data 21. For a review of such techniques and more recent progress, see [3].

The tagging of heavy hadrons in the jet offers a unique possibility of studying the hadronic structure inside the jet. By considering jets where the leading hadron is tagged, a clear connection can be made with perturbative QCD. At the parton level, the tagging of a heavy hadron corresponds to the tagging of a heavy-flavor quark. Experimentally, the presence of a $D$ or $B$ meson is inferred through its decay products. The importance of heavy flavor tagging has been clearly demonstrated in the analysis that led to the top quark discovery [1]. In the future, heavy flavor tagging will continue to be an important analysis tool. It will provide more detailed information about the event, and test of the underlying QCD theory.

If one demands the presence of a charm quark in the jet recoiling against a $W$-boson, the signal is directly sensitive to the strange quark distribution function in the proton, at a scale of the order of the $W$-mass.

Replacing the charm quark by a bottom quark, one could use this process as an alternative calibration of the $b$-quark tagging efficiency. This will be useful at luminosities achieved by the Main Injector. However, by far the dominant contribution to $W+$ bottom production is due to $W+b \bar{b}$ production, where the heavy quark pair is produced by gluon splitting. Here the inclusion of the gluon to $B$ meson fragmentation function is probably more important than the inclusion of NLO effects to the $W$ +bottom process, which implies that this reaction can constrain this fragmentation function.

In this report, which is based on Ref. [7], we present the calculation of the QCD corrections up to $O\left(\alpha_{s}^{2}\right)$ of the process $p \bar{p} \rightarrow W+Q$ where $Q$ is an heavy quark, keeping the mass of the heavy quark explicit, and the final state fully exclusive.

*Talk presented by E. Laenen. 


\section{METHOD}

The method we used to calculate the $O\left(\alpha_{s}^{2}\right)$ QCD corrections to $p \bar{p} \rightarrow W+Q$ is a generalization [8] of the phase space slicing method of Ref. [9] to include massive quarks.

The leading order ( $\mathrm{LO}$ ) calculation involves the simple subprocess $q+g \rightarrow W+Q$, where $q$ is a light quark and $g$ a gluon, at tree level.

The virtual corrections consist of the interference between the lowest order diagrams and their one-loop corrections. The integrals over the loop momenta were performed in $d=4-2 \epsilon$ dimensions. Ultraviolet singularities were absorbed through mass (on-shell scheme) and couplingconstant (modified $\overline{\mathrm{MS}}$ scheme 10$]$ ) renormalization. The remaining soft and collinear singularities, appearing as $1 / \epsilon^{2}$ and $1 / \epsilon$ poles, factorize into a universal factor multiplying the Born cross section.

The real corrections consist of the contributions from all the subprocesses $i j \rightarrow W Q k$, where $i, j, k$ are massless partons, and the subprocess ij $\rightarrow W Q \bar{Q}$ [11]. Some of these contributions exhibit soft and/or collinear singularities. We do not consider diagrams where the heavy quark is in the initial state. The method we used to isolate the singularities consists of slicing up the phase space, using a cut-off $s_{\min }$ and color-ordered subamplitudes, into a hard region $\left(s_{\min }<2 p_{r} \cdot p_{s}\right.$ for any $r, s)$, containing no singularities, and a complementary region in which the final state parton is either soft or emitted collinearly with one of the initial state partons. In the hard phase space region, one can work in four dimensions and perform the phase space integration numerically. In the soft and collinear region, the integration is done analytically in $d$ dimensions using soft and collinear approximations, which are valid in the limit that $s_{\min }$ is small. The cross section in this region again factorizes into a universal factor multiplying the Born cross section. The initial state collinear singularities are factorized into parton distribution functions in the $\overline{\mathrm{MS}}$ scheme, using the formalism of crossing functions [1].

Note that the process $i j \rightarrow W \bar{Q} \bar{Q}$ is quite different from the other subprocesses: the heavy quark does not originate from the $W$ vertex, and it is independent of $s_{\min }$, because it is free from singularities.

Adding the real and virtual corrections leads to the cancellation of all remaining singularities. We checked gauge invariance for both the virtual and real corrections. Finally, we constructed a Monte Carlo program for the present process, including these corrections.

Before showing any numerical results, we first list here the default choices we made for parameters and cuts in producing the results of this paper. Any deviation from these choices will be indicated explicitly. For the case of charm (bottom) we assumed three (four) light flavors. We used both at LO and NLO the CTEQ3M 12 set of parton distribution functions, and a twoloop running coupling constant with $\Lambda_{\mathrm{QCD}}^{(2,4)}$ the value supplied with the CTEQ3M set (0.239 $\mathrm{GeV}$ ). We implemented continuity across heavy flavor thresholds 13 using the parametrization of Ref. [14. We used the Snowmass convention 15] for the definition of a jet. Our conditions on the transverse energy and pseudorapidity of the jet were $E_{T}$ (jet) $>10 \mathrm{GeV},\left|\eta_{j e t}\right|<3$, and no cuts on the W. We took a jet cone size of $\Delta R=0.7$, the $W$ mass equal to $m_{W}=80.23 \mathrm{GeV}$, and the heavy quark mass $m$ equal to $1.7 \mathrm{GeV}$ for charm and $5 \mathrm{GeV}$ for bottom. We used $V_{c s}=0.97$ and $V_{c d}=0.22$ for the relevant Cabibbo-KobayashiMaskawa matrix elements. We chose the factorization scale equal to the renormalization scale and denote it by $\mu$, taking $\mu=m_{W}$. At least one heavy quark was required to be inside of the jet, with the sign of its electric charge correlated with the $W$ charge, as in the LO case.

We verified the independence of the calculation on the arbitrary parameter $s_{\min }$. The results given in the remainder of this report are averaged over $s_{\text {min }}$ between 1 and $10 \mathrm{GeV}$.

We further found the scale dependence of the inclusive cross section to be somewhat reduced by the inclusion of the NLO corrections.

\section{STRANGE QUARK DISTRIBUTION FUNCTION}

We first discuss the effect that the inclusion of the NLO QCD corrections to $W+$ charm-tagged jet 
Table 1

The $W+$ charm-tagged one-jet inclusive cross section in pb for LO, $W+Q \bar{Q}$, and NLO (including the $W+Q \bar{Q}$ contribution) using different sets of parton distribution functions. The statistical uncertainty from the Monte-Carlo integration is less than $1 \%$.

\begin{tabular}{lcccc}
\hline set & mass $(\mathrm{GeV})$ & LO & $W Q Q$ & NLO \\
CTEQ1M & $m_{c}=1.7$ & 96 & 20 & 161 \\
MRSD0' & $m_{c}=1.7$ & 81 & 20 & 138 \\
CTEQ3M & $m_{c}=1.7$ & 83 & 20 & 141 \\
CTEQ3M & $m_{b}=5.0$ & 0.17 & 9.09 & 9.33
\end{tabular}

production has on constraining the strange quark distribution function $s(x, \mu)$ in the proton. Here $x$ is the momentum fraction of the strange quark in the proton, and $\mu$ is the factorization scale. See Ref. [5] for an extensive study of this issue using the shower Monte-Carlo program PYTHIA [6].

In Table 1 we give the NLO cross section for the parton distribution function sets CTEQ1M [16 and MRSD0' 177. The MRSD0' set derives its strange quark distribution from di-muon data, whereas the CTEQ1M set uses DIS data. At low $\mu^{2}$ and low $x$ the difference is as much as a factor of two. Also shown is the result obtained with the more recent CTEQ3M set, which uses the same assumption about the strange quark distribution as MRSD0'. Comparing the CTEQ3M and MRSD0' sets, we see that the difference due to using more recent data sets in the global fit for the parton distribution functions is small. This is also reflected in the cross section for $W+c \bar{c}$, which is the same for all three sets. We can conclude that the difference between CTEQ1M and MRSD0' of $15.4 \%$ is due to the strange quark distribution function. This difference becomes $14.5 \%$ when one includes the $W+b$ background (9 pb, almost all of it coming from the gluon splitting contribution, see Table 1), and assuming conservatively that each bottom quark is mistagged as a charm quark. This shows that the conclusions reached in Ref. [5] are still valid at NLO. In both the NLO calculation and the PYTHIA analysis about $50 \%$ of the contributions are initiated by strange quarks. One major difference is that PYTHIA suggests that the gluon splitting contributes about $35 \%$, whereas in the NLO calculation it is only about $15 \%$. This number is however quite sensitive to the choice of scale $\mu$ in the gluon splitting contribution.

\section{MASS DEPENDENCE OF JET PRO- DUCTION}

Next we compare $W+$ untagged jet production? [1] and $W+$ charm-tagged jet production. In particular, we study the jet transverse energy $\left(E_{T}(\right.$ jet $\left.)\right)$ distribution. In Fig. 1 we show the ratio of the charm-tagged jet over the untagged jet $E_{T}$ (jet)-distribution for the LO and NLO cases. At LO the charm-tagged jet is simply represented by a charm quark. The ratio in Fig. 1 1 has a characteristic shape which can readily be understood at tree level. At low $E_{T}$ (jet) the charm-tagged jet rate is suppressed relative to the untagged-jet rate due to its fermionic final state. The untaggedjet rate is dominated by the gluonic final state which has a soft singularity, that is absent for the fermionic final state. At high $E_{T}$ (jet) we again observe a relative suppression of the charmtagged jet because at LO this process has a gluon in the initial state. At high $E_{T}$ (jet) the dominant scattering in the untagged-jet rate is due to quark-antiquark collisions, again favoring the gluonic final state. Clearly, apart from an approximate overall $K$-factor, the NLO cross section retains these features. Also shown in Fig. 1 is the $W+c \bar{c}$ contribution. At low $E_{T}$ (jet) its suppression is more pronounced due to the charm quark pair production threshold. At high $E_{T}$ (jet) there is no suppression for this process because it is instigated by a quark-antiquark collision.

We now turn to the mass dependence of the cross section. We will show that there are im-

2 For the untagged process we take five massless quark flavors. 


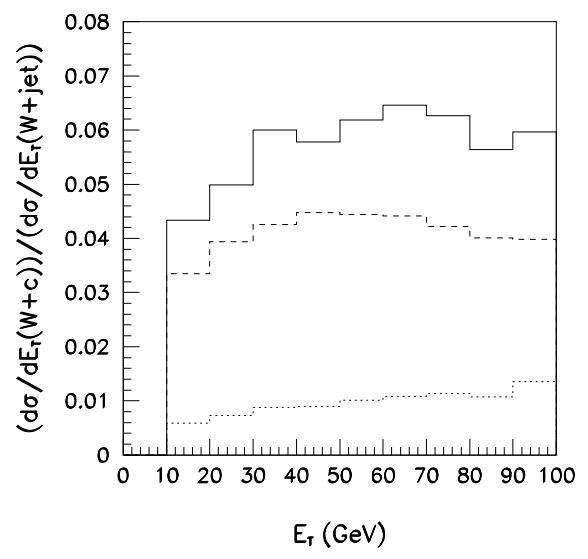

Figure 1. Ratio of $W+$ charm-tagged inclusive one-jet production over $W+$ inclusive untagged one-jet production as function of the jet transverse energy. The solid line is the NLO ratio and the dashed line the LO ratio. The $W+c \bar{c}$ contribution to the NLO ratio is also shown (dotted line).

portant mass effects at NLO, especially when we look in detail at the tagged jets. (At LO one may safely take the mass to zero; the answer does not change.)

Let us first consider the $W+Q \bar{Q}$ contribution. Because the mass of the heavy quark regulates the collinear singularity, it is expected that the strongest mass dependence will come from the collinear region. In this region the cross section factorizes into the cross section for $W+$ gluon production multiplied by a universal factor. After integration over the invariant mass of the heavy quark pair we find that the mass dependent part of this universal factor has the following form:

$\alpha_{s} \frac{N_{c}}{8 \pi} P_{q \bar{q} \rightarrow g}(z) \ln \left(\frac{M^{2}}{m^{2}}\right) d z$

where $M$ is the upper limit of the heavy quark pair invariant mass defining the collinear region, and $P_{q \bar{q} \rightarrow g}(z)$ is the massless Altarelli-Parisi [18] splitting function:

$P_{q \bar{q} \rightarrow g}(z)=\frac{2}{N_{c}}\left(z^{2}+(1-z)^{2}\right)$.
We chose the following definition of $z$

$$
z=\frac{E+P_{\|}}{E_{j e t}+P_{j e t}}
$$

where $E$ and $P_{\|}$are the heavy quark energy and momentum projected on the jet direction, and $E_{j e t}$ and $P_{j e t}$ are the jet energy and momentum. Other choices, such as $z=E_{T}(Q) / E_{T}$ (jet), do not change any of the conclusions in what follows. In the strictly collinear limit $M$ is much smaller than the energy of the gluon, but in a leading logarithmic approximation one may take
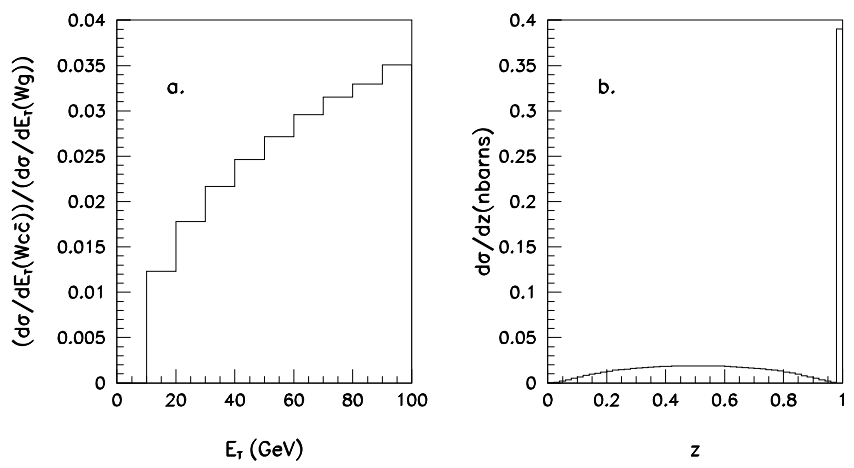

Figure 2. a) Ratio of the $W+c \bar{c}$ component of the charm-tagged one-jet inclusive cross section to the $W+$ gluon cross section, as a function of the jet tranverse energy. b) The $z$-distribution of the $W+c \bar{c}$ component.

$M$ to be of the order of $E_{T}$ (jet). The behavior of Eq. (1) can be seen qualitatively in Fig. 2a, where the ratio of the $W+c \bar{c}$ cross section over the $W+$ gluon cross section is shown as a function of the transverse energy of the jet. One can see an approximate logarithmic enhancement with increasing $E_{T}$, as predicted by the leading logarithmic approximation. However the $z$-distribution, plotted in Fig. 2b, does not conform with the $z$ dependence in Eq. (11). First, the peak at $z=1$ is due to events where the $\bar{Q}$ is not inside of the jet, such that the whole jet is formed by the lone $Q$. Second, the cross section is suppressed near $z=0$ and $z=1$ (excluding the peak) due to terms in the collinear region that depend strongly on $z$, 
but not on $M$. One way to enhance the effect of the leading logarithm term of Eq. (11) in the $z$ distribution is to lower the mass of the heavy quark in our calculation. This is done in Fig. 3a, where we show the $z$-distribution for the case $m=0.01$ $\mathrm{GeV}$. Clearly it now resembles the functional form of Eq. (2) much closer.

The $\ln \left(m^{2}\right)$ term in Eq. (11) diverges in the limit of vanishing quark mass, and is not cancelled by any other contribution [. In principle, any observable should be "collinear safe", i.e. if the mass is taken to zero, the observable should be finite and approach the massless result. This is needed to describe situations where the relevant scale is much larger than the heavy quark mass. In the present case we are dealing with a final state divergence in $\ln \left(m^{2}\right)$ that should be factorized into the fragmentation function of a gluon into a heavy hadron. The evolution of fragmentation functions will then resum the large logarithms $\ln \left(E_{T}^{2}\right)$. This problem is the final state version of the problem of heavy quark distribution functions [19]. It will be discussed in more detail in $[8$. Some studies in this regard were done in Ref. [20]. Here, we simply keep the mass finite. Let us now turn to
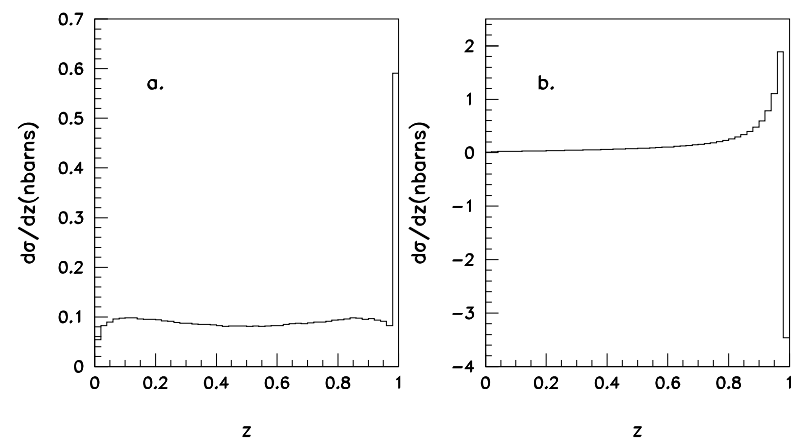

Figure 3. The $z$-distribution of the charm-tagged one-jet inclusive cross section, with $m=0.01$ GeV. a) $W c \bar{c}$ component. b) Total contribution minus the $W c \bar{c}$ component.

\footnotetext{
${ }^{3}$ In the $W+1$ jet calculation, this singularity is cancelled by a companion collinear singularity in the quark-loop correction to the outgoing gluon in the Born diagram. In our calculation this diagram is not present.
}

the NLO single charm contribution (excluding the $W+c \bar{c}$ contribution). In the collinear region, the cross section again factorizes, leading, after integration over the invariant mass of the collinear partons, to the universal factor:

$\alpha_{s} \frac{N_{c}}{8 \pi} P_{q g \rightarrow q}(z) \ln \left(\frac{M^{2}}{m^{2}}\right) d z$

where $P_{q g \rightarrow q}(z)$ is the splitting function:

$$
\begin{array}{r}
P_{q g \rightarrow q}(z)=\lim _{\delta \rightarrow 0} 2\left(1-\frac{1}{N_{c}^{2}}\right)\left(\left(\frac{1+z^{2}}{1-z}\right) \times\right. \\
\left.\theta(1-z-\delta)+\left(\frac{3}{2}+2 \ln \delta\right) \delta(1-z)\right) .
\end{array}
$$

In Fig. 6b we show for the single charm quark contribution the $z$-distribution, with the contribution of Eq. (位) enhanced by taking $m=0.01$ $\mathrm{GeV}$. Note that it resembles the functional form in Eq. (5). From Eq. (5) one derives

$$
\int_{0}^{1} P_{q g \rightarrow q}(z) d z=0 \text {, }
$$

which must hold for the probability to find a quark in a quark of the same flavor to be one [18]. From Eqs. (6) and (14) we can now make the important observation that as long as the cuts on the heavy quark-tagged jet are such that all $z$-values are allowed to contribute, there are no large logarithms $\ln \left(E_{T}^{2} / m^{2}\right)$. However, if the cuts are such that the $z$-integration is restricted, or convoluted with a $z$-dependent function, some $\ln \left(E_{T}^{2} / m^{2}\right)$ terms will remain. An example of such a convolution is the $E_{T}$ distribution of the heavy quark itself. All this is illustrated in Fig. 四a, where two cases of $z$-restrictions $(z>0.9$ and $z<0.9)$ are plotted in addition to the all- $z$ case. Note that for the all- $z$ case the ratio is indeed essentially independent of $E_{T}$ (jet), but that for the $z$-restricted cases the logarithmic dependence is apparent. For completeness we show in Fig. Ab the $z$-distribution for the single charm contribution for $m=1.7 \mathrm{GeV}$.

Thus care must be taken when calculating tagged cross sections in determining whether or not there are large logarithms present due to restrictions on the $z$-integration. Such restrictions would also necessitate the introduction of the appropriate fragmentation function to absorb the $\ln \left(m^{2}\right)$ terms, as in the $W Q \bar{Q}$ case. 

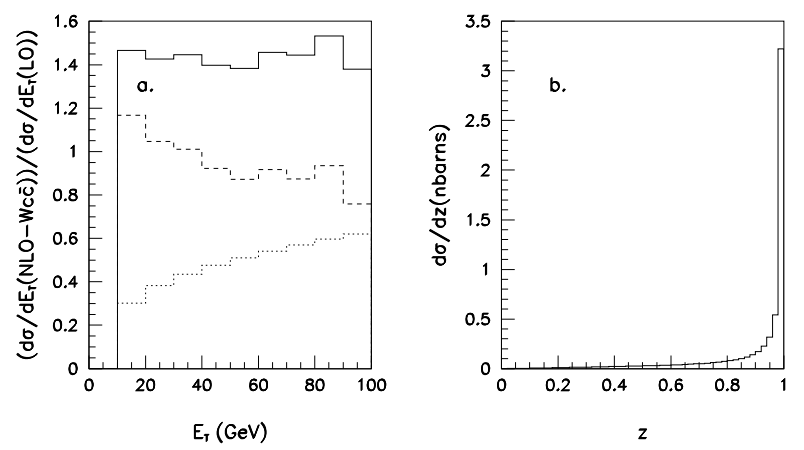

Figure 4 a) $E_{T}$ distribution ratio of NLO charm-tagged one-jet inclusive cross section to LO one. Solid line has no restrictions on the $z$ integration, the dotted and dashed line have restrictions of $z<0.9$ and $z>0.9$ respectively. b) $z$-distribution of NLO charm-tagged one-jet inclusive cross section. The $W+c \bar{c}$ component is not included in these plots.

Note that for the example of the jet transverse energy distribution given in Fig. 4 there is no constraint on the $z$-integration, so that the only final state $\ln \left(m^{2}\right)$ term arises from the $W c \bar{c}$ contribution.

\section{CONCLUSIONS}

We have presented the calculation of the $O\left(\alpha_{s}\right)$ corrections to the reaction $p \bar{p} \rightarrow W+Q$. We found that the inclusion of the NLO corrections does not change the conclusions of Ref. [5] about constraining the strange quark distribution function using $W+$ charm-tagged jet events at the Tevatron. However, using our NLO calculation, this procedure can constrain the NLO strange quark distribution function, once a reasonable data sample is collected. We studied the $E_{T}$ distribution of the jet containing the heavy quark and the mass dependence of the cross section, and noted the need to include the heavy hadron fragmentation functions.

\section{REFERENCES}

1. W.T. Giele, E.W.N. Glover, and D.A. Kosower, Nucl. Phys. B403 (1993) 633.
2. S. Abachi et al (D0 collaboration), Phys. Rev. Lett. 75 (1995) 3226.

3. Z. Kunszt, these proceedings; D.A. Kosower, these proceedings; S. Catani and M. Seymour, these proceedings and hep-ph/9605323.

4. F. Abe et al (CDF collaboration), Phys. Rev. Lett. 74 (1995) 2626; S. Abachi et al (D0 collaboration), Phys. Rev. Lett. 74 (1995) 2632.

5. U. Baur, F. Halzen, S. Keller, M.L. Mangano and K. Riesselmann, Phys. Lett. B318 (1993) 544.

6. H.-U. Bengtsson and T. Sjöstrand, Comp. Phys. Comm. 46 (1987) 43; T. Sjöstrand, preprint CERN-TH.6488/92 .

7. W.T. Giele, S. Keller and E. Laenen, Phys. Lett. 372B (1996) 141.

8. S. Keller and E. Laenen, preprint in preparation.

9. W.T. Giele and E.W.N. Glover, Phys. Rev. D46 (1992) 1980.

10. J.C. Collins, F. Wilczek, and A. Zee, Phys. Rev. D18 (1978) 242;

11. Z. Kunszt, Nucl. Phys. B247 (1984) 339; M.L. Mangano, Nucl. Phys. B405 (1993) 536.

12. H.L. Lai et al, Phys. Rev. D51 (1995) 4763.

13. J.C. Collins and W-K. Tung Nucl. Phys. B278 (1986) 934.

14. G. Altarelli, M. Diemoz, G. Martinelli and P. Nason, Nucl. Phys. B308 (1988) 724.

15. J.E. Huth et al, in Proceedings of the Snowmass Workshop, High Energy Physics in the 1990's, Snowmass, Colorado, July 1990, p. 134.

16. J. Botts et al, Phys. Lett. 304B (1993) 159.

17. A.D. Martin, R.G. Roberts and W.J. Stirling, Phys. Lett. 306B (1993) 145.

18. G. Altarelli and G. Parisi, Nucl. Phys. B126 (1977) 298.

19. M.A.G. Aivazis, J.C. Collins, F.I. Olness and W-K. Tung, Phys. Rev. D50 (1994) 3102.

20. M. Cacciari and M. Greco, Nucl. Phys. B421 (1994) 530; B. Kniehl, M. Krämer, G. Kramer and M. Spira, Phys. Lett. 356B (1995) 539; M. Cacciari and M. Greco preprint DESY 95103. 